\title{
Achyranthes bidentata polypeptide alleviates neurotoxicity of lipopolysaccharide-activated microglia via PI3K/Akt dependent NOX2/ROS pathway
}

\author{
Yitong Wang, Xiangyu Ge, Shu Yu, Qiong Cheng
}

Key Laboratory of Neuroregeneration of Jiangsu and Ministry of Education, Co-innovation Center of Neuroregeneration, NMPA Key Laboratory for Research and Evaluation of Tissue Engineering Technology Products, Nantong University, Nantong, China

Contributions: (I) Conception and design: Q Cheng; (II) Administrative support: Q Cheng; (III) Provision of study materials or patients: Q Cheng; (IV) Collection and assembly of data: All authors; (V) Data analysis and interpretation: All authors; (VI) Manuscript writing: All authors; (VII) Final approval of manuscript: All authors.

Correspondence to: Dr. Qiong Cheng. Key Laboratory of Neuroregeneration of Jiangsu and Ministry of Education, Co-innovation Center of Neuroregeneration, NMPA Key Laboratory for Research and Evaluation of Tissue Engineering Technology Products, Nantong University, 19 Qixiu Road, Nantong 226001, China. Email: cq1981@ntu.edu.cn.

Background: Achyranthes bidentata polypeptide fraction k (ABPPk) has been shown to protect ischemic stroke and Parkinson's disease, and can inhibit neuroinflammation in lipopolysaccharide (LPS)-activated BV2 microglia. However, the effect of ABPPk responsible for alleviating microglial neurotoxicity remains unknown.

Methods: Primary microglia were cultured to investigate the effect of ABPPk on LPS-induced neuroinflammation. Microglia conditioned medium (MCM) was collected to stimulate primary cortical neurons and then the neuronal viability, lactate dehydrogenase $(\mathrm{LDH})$ release, intracellular calcium influx, mitochondria membrane potential (MMP) were assessed, respectively. Postnatal day 5 Sprague-Dawley rat pups were intracerebral injected with LPS to establish an LPS-induced brain injury model. Double immunohistochemical staining for NeuN and Iba1 was performed to evaluate the effects of ABPPk on LPSinduced neuronal damage and microglial activation. TUNEL assay was conducted to detect cell apoptosis in LPS-injected brain. The effect of ABPPk on LPS-induced NADPH oxidase 2 (NOX2) expression and reactive oxygen species (ROS) production as well as the phosphorylation of protein kinase B (Akt) was detected. Moreover, LY294002 (a specific PI3K inhibitor) and SC79 (a specific Akt activator) were used to further reveal the underlying mechanism.

Results: ABPPk pretreatment inhibited LPS-induced NLRP3 and cleaved caspase 1 expressions as well as the mRNA levels of IL-1 $\beta$ and IL-18. Moreover, ABPPk inhibited glutamate release from LPS-activated microglia in a concentration-dependent manner. MCM stimulation resulted in characteristic neuronal toxicity including neuronal viability decrease, $\mathrm{LDH}$ release increase, calcium overload, and MMP drop. However, ABPPk pretreatment on microglia reduced the neurotoxicity of MCM. LPS intracerebral injection led to neuronal damage, microglial activation and cell apoptosis in the brain, while ABPPk preadministration significantly inhibited LPS-induced microglial activation and alleviated the brain injury. ABPPk pretreatment inhibited NOX2 expression and ROS production in LPS-activated primary microglia. Signaling pathway analysis showed that $\mathrm{ABPPk}$ promoted the phosphorylation of Akt in microglia and inhibited LPSupregulated NOX2 expression, ROS production, and glutamate release, which can be eliminated by pharmacological inhibition of PI3K. Specific Akt activator could inhibit LPS-induced NOX2 expression, ROS production and glutamate release.

Conclusions: The present results suggested that ABPPk could alleviate neurotoxicity of LPS-activated microglia via PI3K/Akt dependent NOX2/ROS pathway. 
Keywords: Achyranthes bidentata polypeptide; microglia; glutamate release; excitatory neurotoxicity

Submitted Jul 16, 2021. Accepted for publication Oct 02, 2021.

doi: $10.21037 / \mathrm{atm}-21-4027$

View this article at: https://dx.doi.org/10.21037/atm-21-4027

\section{Introduction}

Achyranthes bidentata (A. bidentata) is a famous traditional Chinese medicine, with the effect of dissipating blood stasis, nourishing liver and kidney, and strengthening muscles and bones $(1,2)$. A . bidentata contains a variety of bioactive components, including saponins, polysaccharides, and polypeptides. Our research team carried out a series of studies on the aqueous extract of $A$. bidentata and $A$. bidentata polypeptides (ABPP) and showed that the aqueous extract of $A$. bidentata could combat glutamate-induced neurotoxicity in hippocampal neurons (3), ABPP could resist excitatory toxic neuronal injury through inhibiting the $\mathrm{N}$-methyl-D-aspartate (NMDA) receptors $2 \mathrm{~B}$ subunit (4), while further purification and analysis of ABPP showed that the fraction $\mathrm{k}$ (ABPPk) had the best neuroprotective effect in cerebral ischemic model and Parkinson's disease model (5-7). In addition, recent studies have shown that ABPPk could reduce the production of nitric oxide (NO), prostaglandin E2 (PGE2), tumor necrosis factor $\alpha$ (TNF- $\alpha$ ), and interleukin 6 (IL-6) in lipopolysaccharide (LPS)-activated BV2 microglia (8). However, the mechanisms by which ABPPk alleviate the neurotoxicity of activated microglia remain unclear.

As innate immune cells in the brain, microglia are usually the first responders to brain injury (9). Microglia activated by injury rapidly undergo morphological changes, from ramified to amoeba-like morphology and serve a dualistic role performed by different polarization phenotypes in response to different microenvironmental cues (10). On the one hand, alternatively activated M2 microglia produce chemokines to recruit additional cells to clear pathogens and cell debris, resolve local inflammation, and release nutritional factors to promote brain recovery. On the other hand, classically activated M1 microglia produce proinflammatory cytokines, reactive oxygen species (ROS), excitatory amino acid and so on to magnify tissue damage and hinder central nervous system repair (11-13). Studies in cultured neurons demonstrated that most of microgliaderived inflammatory cytokines, superoxide, nitric oxide (NO), etc. can damage neurons and lead to necrosis or apoptosis through a cascade reaction. Excitatory amino acid, especially glutamate, released by activated microglia can cause excitatory neurotoxicity leading directly to neuronal death, which is thought to be the primary cause of neurotoxicity of microglia (14-18). LPS stimulation triggered oxidative stress and respiratory burst in microglia, leading to continuous release of glutamate, which may be the key to the conversion of oxidative stress into excitatory toxic stress in neuroinflammation $(19,20)$, and active ingredients or compounds that can interfere with these events, including the regulation of glutamate release from activated microglia, may be useful in the treatment of neuroinflammatory diseases (21). We speculate that intervention with $\mathrm{ABPPk}$ in microglia would reduce its neurotoxicity. In this study, we investigated the effect of ABPPk on the neurotoxicity of LPS-stimulated microglia. In vitro, the toxicity of LPS-activated microglia conditioned medium (MCM) on neurons was evaluated from the aspects of cell viability, lactate dehydrogenase (LDH ) release, calcium influx and mitochondrial membrane potential (MMP) changes. In vivo, the effect of ABPPk on LPSinduced neuronal death, microglial activation, and cell apoptosis was investigated, and the mechanism of ABPPk alleviating microglial neurotoxicity was explored. We present the following article in accordance with the MDAR reporting checklist (available at https://dx.doi.org/10.21037/ atm-21-4027).

\section{Methods}

\section{Reagents and antibodies}

LPS (Escherichia coli O111:B4) and poly-L-lysine (PLL) were purchased from Sigma (St. Louis, USA) and CCK-8 was purchased from Dojindo (Kumamoto, Japan). Amplex ${ }^{\mathrm{TM}}$ Red Glutamic Acid/Glutamate Oxidase Assay Kit (\#A-12221) was purchased from Invitrogen (Carlsbad, USA), while 2,7-Dichlorofluorescein diacetate (DCFH-DA) kit (\#S0033), 5,5',6,6'-tetrachloro-1,1',3,3'tetraethyl-imidacarbocyanine (JC-1) kit (\#C2006), lactate dehydrogenase (LDH) cytotoxicity assay kit (\#C0017), TUNEL apoptosis assay kit (\#C1086) as well as SC79 (\#SF2730) were all purchased from Beyotime (Shanghai, 
China). IL-1 $\beta$ ELISA kit (\#BE45111) was obtained from IBL International (Hamburg, Germany). LY294002 (\#9901S) was purchased from Cell Signaling Technology (Danvers, USA). Dulbecco's modified Eagle's medium (DMEM), fetal bovine serum (FBS), Neurobasal medium, B27, penicillin, and streptomycin were all from Invitrogen (Carlsbad, USA). A protein extraction kit, BCA protein assay kit, and ECL Western Blotting Substrate were all purchased from Thermo Fisher Scientific (Waltham, USA). Antibodies specific for caspase 1 (\#ab207802), NeuN (\#ab104224), and NOX2 gp91phox (\#ab80508) were obtained from Abcam (Cambridge, UK) and antibodies specific for NLRP3 (\#15101S), phosphorylated-Akt (Ser473) (\#4060S), Akt (\#9272S) and $\beta$-actin (\#3700S) were obtained from Cell Signaling Technology (Beverly, USA). Antibody specific for Iba1 (\#019-19741) was obtained from Wako (Kyoto, Japan).

\section{Preparation of $A B P P k$}

The root of $A$. bidentata was purchased from a local Chinese medicine grocer and identified by the pharmacist. ABPPk was isolated from $A$. bidentata and purified by HPLC as previously described $(5,22)$.

\section{Primary cell culture and treatment}

Primary microglia were isolated from the brain of newborn $1 \mathrm{~d}$ C57BL/6 mouse pups using a simple culture procedure with some modifications as reported previously (23). Briefly, the cortical and hippocampal tissues were digested with $0.125 \%$ trypsin at $37^{\circ} \mathrm{C}$ for $15 \mathrm{~min}$, then $1 \mathrm{~mL}$ FBS was added to terminate the digestion. The homogeneous cell suspension was plated in PLL-coated Corning T-75 flasks containing DMEM supplemented with 10\% FBS and antibiotics. The culture medium was changed the next day to remove cell debris. Within 7 days, the astrocytes at the bottom of the flask formed a layer of fused cells, while microglia and some oligodendrocytes grew at the top of the astrocyte layer. On the 8th day of culture, microglial cells were recovered by trypsin proteolysis using $0.05 \%$ trypsin. These resulting cells were purified microglia and were seeded in PLL-coated culture plates at a proper density. To examine the effect of ABPPk on LPS-activated microglia, cells were pretreated with different concentrations of $\mathrm{ABPPk}$ $(0.2,1$, and $5 \mu \mathrm{g} / \mathrm{mL})$ for $30 \mathrm{~min}$ followed by LPS $(1 \mu \mathrm{g} / \mathrm{mL})$ stimulation for $24 \mathrm{~h}$, then the subsequent experiments were performed as designed. The concentration of ABPPk was referred to our previous study (8).
Primary cultures of cortical neurons were obtained from embryonic day 16 (E16) Sprague-Dawley (SD) rats' embryos as previously described (24). In brief, the cortical tissues were harvested and digested enzymatically into cell suspensions. Cells were then re-suspended in DMEM containing $10 \%$ FBS at a proper density and plated onto PLL-coated cell culture plates, followed by incubating in humidified atmosphere of $95 \%$ air and $5 \% \mathrm{CO}_{2}$ at $37^{\circ} \mathrm{C}$. After $4 \mathrm{~h}$, and when cells had attached to the substrate, the medium was exchanged to Neurobasal medium supplemented with $2 \%$ B27 supplement, then re-incubated for 7 days for follow-up experiments.

\section{Quantitative real-time polymerase chain reaction (qRT-PCR)}

Total RNA of microglia was extracted after $24 \mathrm{~h}$ stimulation in the presence or absence of $\mathrm{ABPPk}$ pretreatment using RNeasy Mini Kit (QIAGEN, CA, USA), then cDNA was synthesized from total RNA by reverse transcription using Omniscript RT kit (QIAGEN) as per the manufacturer's instructions. qPCR was carried out in the Stepone RTPCR instrument (Applied Biosystems, CA, USA) using SYBR Green Supermix (Bio-Rad, CA, USA). The housekeeping gene Gapdh was served as an internal control. Primers were designed and synthesized by GENEray Biotechnology (Shanghai, China). The primer sequences were as follows: IL-1 $\beta$, F: GAGAGCATCCAGCTTCAAA, R: T C A T C A T C C C A C G A G T C A ; I L 18, F: AACGAAT C C CA GAC CA GAC, R : AGAGGGTAGACATCCTTCCAT; Gapdh, F: C G TAT T G G G C G C C T G G T C A C C A G ; R : GACCTTGCCCACAGCCTTGGCAGC.

\section{Measurement of extracellular glutamate}

To evaluate the effect of ABPPk on LPS-induced glutamate release, microglia were planted on 96-well plates at a density of $5 \times 10^{5}$ cells $/ \mathrm{mL}$, then pretreated with $0.2 \mu \mathrm{g} / \mathrm{mL}, 1 \mu \mathrm{g} / \mathrm{mL}$ or $5 \mu \mathrm{g} / \mathrm{mL} \mathrm{ABPPk}$ for $30 \mathrm{~min}$ before $1 \mu \mathrm{g} / \mathrm{mL}$ LPS stimulation. After incubation for $24 \mathrm{~h}$, glutamate release was detected by Glutamate assay kit according to the instructions; add $50 \mu \mathrm{L}$ of the Amplex Red regent to each microplate well (10 wells per group), then the cells were incubated for $30 \mathrm{~min}$ at $37^{\circ} \mathrm{C}$ in dark, while the untreated cells served as control. Fluorescence was measured in a microplate reader using the excitation of $530 \mathrm{~nm}$ and emission of $590 \mathrm{~nm}$, and the amount of 
glutamate released $(\mu M)$ was calculated using the standard curve.

\section{Microglia conditioned medium (MCM) system}

MCM was prepared as previously described (25). Microglia were seeded at a density of $5 \times 10^{5}$ cells $/ \mathrm{mL}$ in a 6 -well culture plate, and after the cells were pre-treated with $5 \mu \mathrm{g} / \mathrm{mL} \mathrm{ABPPk}$ for $30 \mathrm{~min}, 1 \mu \mathrm{g} / \mathrm{mL}$ LPS were added in the culture media. After $24 \mathrm{~h}$, the culture media were collected and centrifuged at 1,200 rpm for $5 \mathrm{~min}$ to obtain the supernatant, which served as MCM. LPS stimulation without ABPPk pretreatment made L-MCM, and LPS stimulation after ABPPk pretreatment made A+L-MCM.

\section{Cell viability assay}

Cortical neurons were seeded in a 96-well plate with a density of $1 \times 10^{6}$ cells $/ \mathrm{mL}$ for $7 \mathrm{~d}$, when the medium was removed and washed once with PBS solution. The prepared MCMs were used to stimulate the neurons for $24 \mathrm{~h}$ (10 wells per group), then $10 \mu \mathrm{L}$ CCK- 8 was added to each well and incubated at $37^{\circ} \mathrm{C}$ in a water bath in the dark for $2 \mathrm{~h}$ to detect the cell viability. The absorbance was determined at a wavelength of $450 \mathrm{~nm}$ using a microplate reader (BioTek, Vermont, USA). The data were presented as the percentage of cell viability relative to the control.

\section{Lactate dehydrogenase (LDH) release assay}

Cortical neurons were seeded in a 96-well plate with a density of $1 \times 10^{6}$ cells $/ \mathrm{mL}$ for $7 \mathrm{~d}$, at which time the medium was removed and then washed with PBS once. MCMs were then added for stimulation (10 wells per group), and after cultured for $24 \mathrm{~h}, 150 \mu \mathrm{L}$ PBS-diluted LDH release reagent was added to each well. The plate was then shaken well, incubated for a further $1 \mathrm{~h}$, then centrifuged at 1,000 rpm for $5 \mathrm{~min} ; 120 \mu \mathrm{L}$ supernatant was taken from each well and transferred into a new 96-well plate for determination, where a $60 \mu \mathrm{L} \mathrm{LDH}$ test solution was added to each well before mixing and incubation in the dark at room temperature for $30 \mathrm{~min}$. The absorbance was determined at a wavelength of $490 \mathrm{~nm}$ using a microplate reader (BioTek, Vermont, USA). The data were presented as the percentage of cytotoxicity relative to the control.

\section{Measurement of intracellular $\mathrm{Ca}^{2+}$}

Cortical neurons were planted at a density of $1 \times 10^{6}$ cells/ $\mathrm{mL}$ onto a 96-well plate for $7 \mathrm{~d}$, and after stimulation with different MCMs (6 wells per group), the intracellular $\mathrm{Ca}^{2+}$ concentration was measured with Fluo-4/AM by scanning laser confocal microscopy (Leica TCS SP2, Wetzlar, Germany). The cells were loaded with $5 \mu \mathrm{M}$ Fluo-4/AM in Hank's solution at $37^{\circ} \mathrm{C}$ in the dark for $45 \mathrm{~min}$, then washed gently to remove extracellular Fluo-4/AM dye. Prior to stimulation, the dye-loaded cells were scanned for $100 \mathrm{~s}$ to obtain a basal level of intracellular $\mathrm{Ca}^{2+}$. Fluorescence was monitored every $10 \mathrm{~s}$ over a total period of $900 \mathrm{~s}$ (excitation at $488 \mathrm{~nm}$ and emission at $526 \mathrm{~nm}$ ). The intensity was quantified over randomly 30 single neurons, and the data were expressed as the relative fluorescence intensity over the control.

\section{Detection of mitochondria membrane potential (MMP)}

The MMP $(\Delta \Psi \mathrm{m})$ of cortical neurons was detected with a fluorescent probe JC-1. For fluorescence images, cortical neurons were planted at a density of $1 \times 10^{6}$ cells $/ \mathrm{mL}$ onto PLL-coated slides for $7 \mathrm{~d}$, and after stimulation with MCMs (3 slides per group), cultures were incubated with $2 \mathrm{mM} \mathrm{JC}-1$ at $37^{\circ} \mathrm{C}$ for $15 \mathrm{~min}$. Slides were washed and mounted, and fluorescence images were acquired with a fluorescence microscope (Leica, Wetzlar, Germany). For flow cytometry, cortical neurons were planted at a density of $1 \times 10^{6}$ cells $/ \mathrm{mL}$ onto a PLL-coated 24-well plate for $7 \mathrm{~d}$, and after stimulation with MCMs (3 wells per group), cells were collected and washed in PBS. After being re-suspended and adjusted to the density of $1 \times 10^{6}$ cells $/ \mathrm{mL}$, cells were incubated with $2 \mathrm{mM} \mathrm{JC}-1$ at $37{ }^{\circ} \mathrm{C}$ for $15 \mathrm{~min}$, then washed and analyzed by flow cytometer (BD FACSCalibur, BD Bioscience, San Jose, CA). Photomultiplier settings were adjusted to detect JC-1 aggregate red fluorescent signals on FL2 detector $(585 \mathrm{~nm})$ and JC-1 monomer green fluorescent signals on FL1 detector $(525 \mathrm{~nm})$, then the percentage of living cells and apoptotic cells were calculated, respectively.

\section{Measurement of ROS}

For ROS analysis of primary microglia, cells were seeded in 96-well plates at a density of $5 \times 10^{5}$ cells $/ \mathrm{mL}$, and grouped 
according to the experimental design (10 wells per group). After washing with DMEM, the cells were stained with $10 \mu \mathrm{M}$ DCFH-DA in DMEM for $20 \mathrm{~min}$ at $37^{\circ} \mathrm{C}$ in the dark, then washed twice with DMEM. Fluorescence of each group was detected by fluorescence microplate reader (BioTek, Vermont, USA) with excitation at $484 \mathrm{~nm}$ and emission at $530 \mathrm{~nm}$, and the data were expressed as the percentage of fluorescence intensity to the control.

\section{Animal model}

A total of 18 postnatal 5-day Sprague-Dawley (SD) rat male pups were supplied by the Experimental Animal Center of Nantong University. The study was approved by the Administration Committee of Experimental Animals of Nantong University. All the experimental procedures were performed according to the Chinese Guidelines for the Care and Use of Laboratory Animals. Rat pups were randomly separated into 3 groups (6 animals per group): sham operation (Sham group), LPS injection (LPS group), and ABPPk pre-administration followed by LPS injection (ABPPk group). Model of LPS-induced brain injury was referred to previously reported literatures (26-28). The rat pup was anesthetized by intraperitoneal injection of avertin (1.25\% bromethol, $0.2 \mathrm{~mL} / 10 \mathrm{~g}$ body weight) (AIBI Bio-Tech, Nanjing, China), then a volume of $2 \mu \mathrm{L}$ LPS $(1 \mathrm{mg} / \mathrm{kg})$ was injected using a microsyringe which was inserted perpendicularly through the skull to the brain at the location of $1.0 \mathrm{~mm}$ posterior and $1.0 \mathrm{~mm}$ left to the bregma, and $2.0 \mathrm{~mm}$ deep in a stereotaxic apparatus (RWD 68019, RWD, Shenzhen, China). Rat pups in the ABPPk group were first administrated with $2.5 \mathrm{mg} / \mathrm{kg}$ ABPPk (total volume of $2 \mu \mathrm{L}$ ) at the same location, and then injected with $2 \mu \mathrm{L}$ LPS $(1 \mathrm{mg} / \mathrm{kg}) 10 \mathrm{~min}$ later. Rat pups in the Sham group only exposed the skulls, but no injections were given. All animals survived the intracerebral injection and no one was excluded.

\section{Immunofluorescence staining}

Six hours after LPS injection, three rat pups of each group were sacrificed by transcardiac perfusion with saline followed by $4 \%$ paraformaldehyde (PFA) for brain section preparation. The brain was removed and postfixed overnight in $4 \% \mathrm{PFA}$ at $4{ }^{\circ} \mathrm{C}$, followed by gradient dehydration with sucrose solution. Coronal frozen brain sections at $10 \mu \mathrm{m}$ of thickness were prepared in a cryostat for immunofluorescence labeling. After blocking with $0.1 \%$ Triton $\mathrm{X}-100$ and $5 \%$ normal goat serum at room temperature for $1 \mathrm{~h}$, the sections were then incubated with mouse anti-NeuN (diluted 1:600) and rabbit antiIba1 (diluted $1: 300$ ) overnight at $4{ }^{\circ} \mathrm{C}$. After washing with PBS, the slices were incubated with Alexa Fluor 555 or 488 -conjugated secondary antibodies (goat antimouse, diluted 1:2,000; goat anti-rabbit, diluted 1:2,000, Molecular Probes, USA) in the dark at room temperature for $1 \mathrm{~h}$. The slides were coverslipped with mounting media containing DAPI (Vector, USA) then photographed under a fluorescence microscope. The number of NeuN positive and Iba1 positive cells were counted by a blind investigator.

\section{Terminal deoxynucleoitidyl transferase-mediated dUTP Nick-end labeling (TUNEL) assay}

TUNEL assay kit was used to evaluate the apoptosis of cells in the brain $6 \mathrm{~h}$ after LPS injection. The frozen brain sections were washed with PBS twice for 10 min each time, then incubated with $0.5 \%$ Triton $\mathrm{X}-100$ at room temperature for $5 \mathrm{~min}$. Add $50 \mu \mathrm{L}$ TUNEL detection solution to each section, and incubate at $37^{\circ} \mathrm{C}$ for $1 \mathrm{~h}$ in the dark. Cover the slices with cling film to prevent evaporation of the TUNEL detection solution. After washing with PBS, slides were coverslipped with DAPI mounting media and photographed under the fluorescence microscope. The number of apoptotic cells were counted by a blind investigator and presented as TUNEL-positive cells $/ \mathrm{mm}^{2}$.

\section{Enzyme-linked immunosorbent assay (ELISA)}

Six hours after LPS injection, three rat pups of each group were sacrificed and the brains were removed quickly. After washing with pre-cooled saline, the brains were dried with filter paper and weighed. Pre-cooled homogenizing medium was added at a weight/volume ratio of $1: 10(\mathrm{mg} / \mu \mathrm{L})$, homogenized in ice bath for $3 \mathrm{~min}$, then centrifuged at $15,000 \mathrm{rpm}$ at $4{ }^{\circ} \mathrm{C}$ for $10 \mathrm{~min}$. IL- $1 \beta$ content in the supernatant was detected using the IL- $1 \beta$ ELISA kit, and the absorbance was measured at $450 \mathrm{~nm}$ using a microplate reader (BioTek, Vermont, USA). The content of $\mathrm{IL}-1 \beta$ was calculated per gram of protein $(\mathrm{pg} / \mathrm{g})$.

\section{Western blot analysis}

The whole cell lysates of microglia were extracted and 
collected by centrifugation $\left(13,000 \mathrm{rpm}, 10 \mathrm{~min}, 4^{\circ} \mathrm{C}\right)$, and protein concentrations were determined with Pierce ${ }^{\mathrm{TM}} \mathrm{BCA}$ protein assay. Proteins were electrophoresed on 10\% SDSpolyacrylamide gel and transferred to a PVDF membrane (Millipore, Bedford, MA, USA) which were then blocked in $5 \%$ non-fat milk for $1 \mathrm{~h}$ at room temperature and incubated with the specific primary antibodies (NLRP3, diluted 1:1,000; caspase 1, diluted 1:1,000; p-Akt, diluted 1:2,000; Akt, diluted 1:1,000; NOX2, $1 \mu \mathrm{g} / \mathrm{mL}$; and $\beta$-actin, diluted $1: 1,000)$ overnight at $4{ }^{\circ} \mathrm{C}$. The membranes were incubated with HRP-conjugated secondary antibody for $1 \mathrm{~h}$ at room temperature, and the proteins were then detected with ECL Western Blotting Substrate. Quantitative analysis of Western blot was performed by ImageJ software (NIH Image, Washington, DC, USA).

\section{Statistical analysis}

All data were presented as mean \pm SD using Graph Pad Prism 8.0 (Graph Pad software, San Diego, USA). The results were analyzed by one-way ANOVA analysis followed by Tukey's multiple comparisons test. A P value less than 0.05 was considered statistically significant.

\section{Results}

\section{ABPPk inbibited LPS-induced microglial inflammation}

Our previous study showed that ABPPk inhibited the release of pro-inflammatory factors by LPS-activated BV2 microglia, indicating the inhibitory effect of ABPPk on neuroinflammation. Inflammasomes are important components of the natural immune system and can recruit and activate the pro-inflammatory protease caspase- 1 . The active caspase 1 cleaves the precursors of IL- $1 \beta$ and IL-18 to produce the corresponding mature pro-inflammatory cytokines $(29,30)$. In this study, we found that ABPPk concentration-dependently inhibited LPS-induced primary microglial inflammasome NLRP3 protein and cleaved caspase-1 expression levels, as shown in Figure 1A, and subsequently inhibited IL-1 $\beta$ and IL-18 mRNA levels, as shown in Figure $1 B, 1 C$. In addition, LPS stimulation at $1 \mu \mathrm{g} / \mathrm{mL}$ significantly increased glutamate release from microglia, while ABPPk inhibited LPS-induced glutamate release in a concentration-dependent manner, especially with the concentration of 1 and $5 \mu \mathrm{g} / \mathrm{mL}$ (Figure 1D).

\section{ABPPk pretreatment on microglia alleviated the MCM- induced damage to cortical neurons}

We stimulated cortical neurons with MCMs, then assessed the neuronal damage, and the processing procedure is shown in Figure $2 A$. After L-MCM stimulation for $24 \mathrm{~h}$, a large number of dead cells appeared in the culture medium under the microscope, and most of the remaining neurons were wrinkled and their protrusion collapsed. Although the neurons stimulated by A+L-MCM were partially dead, the morphology of the remaining cells was relatively intact as shown in Figure 2B. CCK-8 results showed that L-MCM decreased the cell viability significantly. However, A+L-MCM showed lower neurotoxicity, and significantly reduced neuronal activity loss compared with the L-MCM stimulation (Figure 2C). LDH assay results showed that L-MCM significantly increased the release of LDH from cortical neurons. In contrast, A+L-MCM resulted in significantly decreased LDH release from neurons compared with L-MCM stimulation (Figure 2D).

\section{ABPPk pretreatment on microglia reduced the MCM- induced calcium influx in neurons}

The excitotoxic dose of glutamate binds to the neuronal glutamate receptors, causing the calcium channel to open, leading to calcium overload and excitotoxic injury (31). To detect whether glutamate released by LPS-activated microglia would cause calcium overload of neurons, Fluo4/AM fluorescent dye was used to detect the calcium influx, and when different MCMs were used to stimulate cortical neurons respectively, changes in the fluorescence intensity of intracellular calcium were monitored by confocal microscope (Figure 3A-3C). Stimulation with $\mathrm{L}-\mathrm{MCM}$ induced a sudden increase in fluorescence intensity, indicating an intracellular $\mathrm{Ca}^{2+}$ influx. The peak fluorescence intensity analysis of each group showed that L-MCM significantly increased the calcium influx in cortical neurons, while A+L-MCM significantly reduced the $\mathrm{Ca}^{2+}$ influx in neurons compared with L-MCM (Figure 3D).

\section{ABPPk pretreatment on microglia reduced the MCM- induced decrease of neuronal MMP}

Mitochondria normally show high membrane potential, at which time a JC-1 fluorescence probe will form aggregates 
A

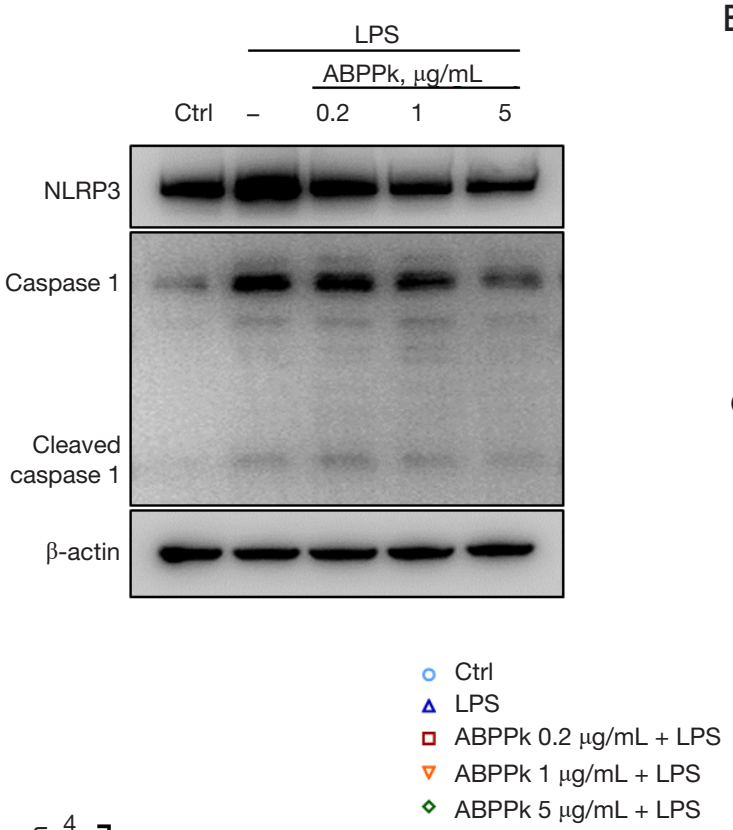

B

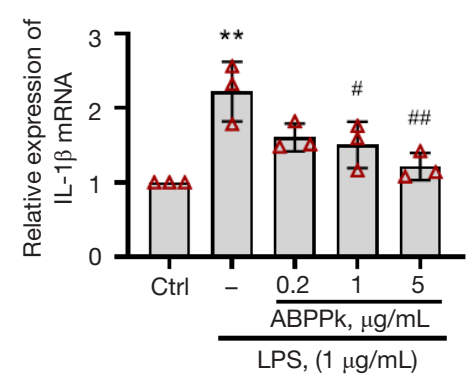

C
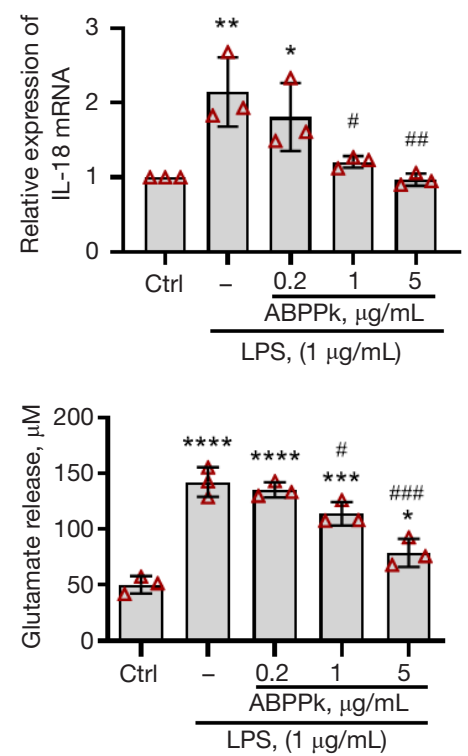

Figure $1 \mathrm{ABPPk}$ inhibited LPS-induced microglial inflammation. (A) Representative Western blot image and gray analysis showing the $\mathrm{NLRP} 3$ and cleaved caspase 1 expressions in different treated microglia as indicated. Data are expressed as mean $\pm \mathrm{SD}(\mathrm{n}=3) .{ }^{* *}, \mathrm{P}<0.01 ;{ }^{* * *}$, $\mathrm{P}<0.001$. (B) Histogram showing the relative expression of IL-1 $\beta$ mRNA measured by qPCR. Data are expressed as mean \pm SD ( $=3$ ). **, $\mathrm{P}<0.01$ vs. Ctrl; \#, $\mathrm{P}<0.05$, \#, $\mathrm{P}<0.01$ vs. LPS. (C) Histogram showing the relative expression of IL-18 mRNA measured by qPCR. Data are expressed as mean $\pm \mathrm{SD}(\mathrm{n}=3)$. *, $\mathrm{P}<0.05$, **, $\mathrm{P}<0.01$ vs. Ctrl; ${ }^{*}, \mathrm{P}<0.05$, \#\#, $\mathrm{P}<0.01$ vs. LPS. (D) Histogram showing the impact of indicated concentrations of ABPPk pretreatment on LPS-induced glutamate release from microglia. Data are expressed as mean $\pm \mathrm{SD}(\mathrm{n}=3)$. * $\mathrm{P}<0.05$, ***, $\mathrm{P}<0.001$, ${ }^{* * *}, \mathrm{P}<0.0001$ vs. Ctrl; ${ }^{\#}, \mathrm{P}<0.05,{ }^{\# \#}, \mathrm{P}<0.001$ vs. LPS. Experiments were triplicated biologically. ABPPk, Achyranthes bidentata polypeptide fraction $\mathrm{k}$; LPS, lipopolysaccharide.

in the mitochondrial matrix, presenting red fluorescence. In the case of dysfunction, MMP would decrease, and JC-1 could not aggregate in the matrix, but would exist as monomers, presenting green fluorescence. In this study, L-MCM prevented the formation of red aggregation in neurons, and a large amount of green monomers could be seen in the culture, which meant L-MCM induced a typical drop of MMP. However, A+L-MCM reduced the decrease of MMP (Figure 4A). Flow cytometry analysis revealed L-MCM stimulated neurons showed early apoptosis and a reduced number of living cells, while A+L-MCM reduced the apoptosis cell percentage significantly (Figure $4 B, 4 C$ ).
These results all suggested that ABPPk pretreatment on microglia alleviated the neurotoxicity of MCM.

\section{ABPPk reduced neuroinflammation induced by LPS injection, protected brain neurons, and reduced cell apoptosis}

At $6 \mathrm{~h}$ after LPS intracerebral injection, NeuN and Iba1 double immunofluorescence staining showed that LPS intracerebral injection resulted in cortical neuronal damage, characterized by the decrease in the number of neurons and the increase in the number of activated microglia, while 
A

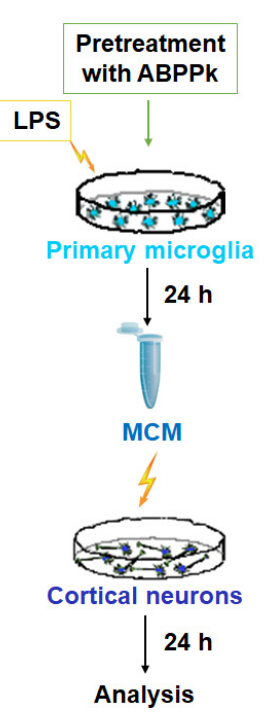

B

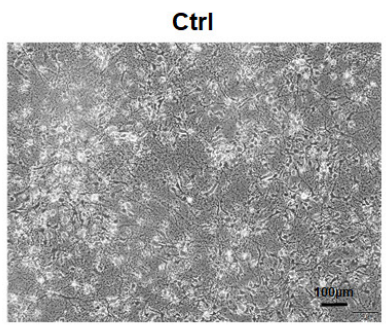

C

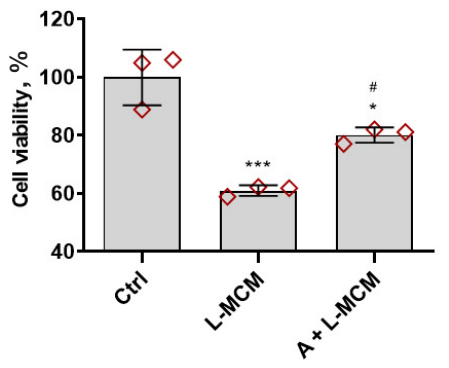

L-MCM

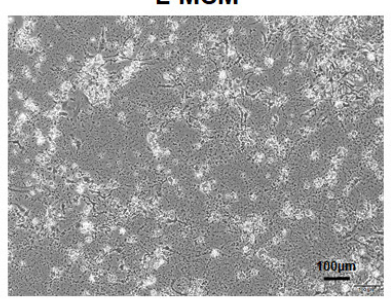

A + L-MCM

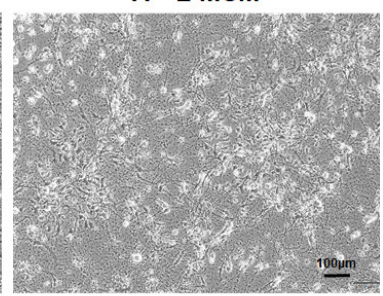

D

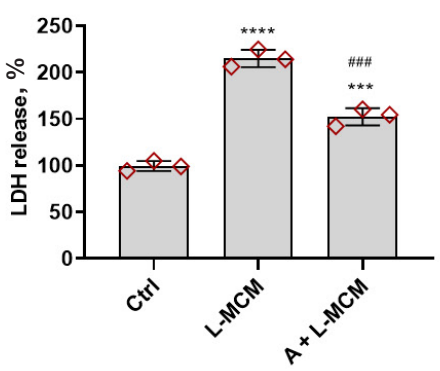

Figure $2 \mathrm{ABPPk}$ alleviated the neurotoxicity of MCM to cortical neurons. (A) The procedure of collecting MCM to stimulate cortical neurons. (B) Typical images of neuron morphology under different stimulus conditions, observed under phase contrast microscope. Scale bar, $100 \mu \mathrm{m}$. (C) Cell viability of cortical neurons stimulated by MCM for $24 \mathrm{~h}$. Data are expressed as mean $\pm \mathrm{SD}(\mathrm{n}=3)$. *, $\mathrm{P}<0.05,{ }^{* * *}, \mathrm{P}<0.001$ vs. Ctrl; ", $\mathrm{P}<0.05$ vs. L-MCM. (D) LDH release of cortical neurons stimulated by MCM for 24 h. Data are expressed as mean \pm SD ( $=3$ ). *** $, \mathrm{P}<0.001,{ }^{* * * *}, \mathrm{P}<0.0001$ vs. Ctrl; ${ }^{\# \#}, \mathrm{P}<0.001$ vs. L-MCM. Experiments were triplicated biologically. ABPPk, Achyranthes bidentata polypeptide fraction k; MCM, microglia conditioned medium; LPS, lipopolysaccharide; LDH, lactate dehydrogenase.

ABPPk suppressed LPS-induced microglial activation significantly (Figure 5A-5C). Although ABPPk didn't significantly reduce LPS-induced neuron loss as compared to LPS group in this study, possibly due to the small sample size, we can still see an improved neuronal loss in the ABPPk group than in the LPS group. ELISA result showed that LPS injection led to a significantly increased level of IL- $1 \beta$ in the brain, while ABPPk preadministration significantly inhibited LPS-induced IL- $1 \beta$ elevation (Figure 5D). TUNEL result showed that a large number of apoptotic cells appeared in the cortex and striatum $6 \mathrm{~h}$ after LPS injection, and ABPPk preadministration could significantly inhibit the cell apoptosis in the brain (Figure 6). These results suggested that $\mathrm{ABPPk}$ inhibited LPS-induced microglial activation and neuroinflammation, as well as alleviated neuronal injury induced by LPS injection in the brain.

\section{ABPPk suppressed LPS-induced NOX2 upregulation and ROS production in microglia}

The production of ROS in activated microglia is a prerequisite for glutamate release. The main source of ROS production is NOX2 gp91phox, which is the main isoform of NADPH oxidase and abundant in microglia $(19,32,33)$. Our result showed that $1 \mu \mathrm{g} / \mathrm{mL}$ LPS stimulation for $24 \mathrm{~h}$ significantly upregulated NOX2 expression in microglia, while ABPPk pretreatment had an inhibitory effect on NOX2 expression induced by LPS (Figure 7A). The DCFH-DA assay was used to measure the production of intracellular ROS production. The result showed that $1 \mu \mathrm{g} / \mathrm{mL}$ LPS stimulation for $24 \mathrm{~h}$ could significantly enhance ROS production in microglia, and $\mathrm{ABPPk}$ pretreatment could inhibit the ROS production in a concentration-dependent manner (Figure 7B).

PI3K/Akt patbway participated in the inbibition of NOX2 by ABPPk and affected the ROS production and glutamate release of microglia

The activation of NOX2 in microglia is controlled by PI3K/ Akt dependent mechanism (34). In this study, microglia were treated with different concentrations of ABPPk for $30 \mathrm{~min}$, then the expression level of phosphorylated-Akt (Ser473) was detected. Western blot result showed that ABPPk promoted the phosphorylation of Akt at Ser473 
A

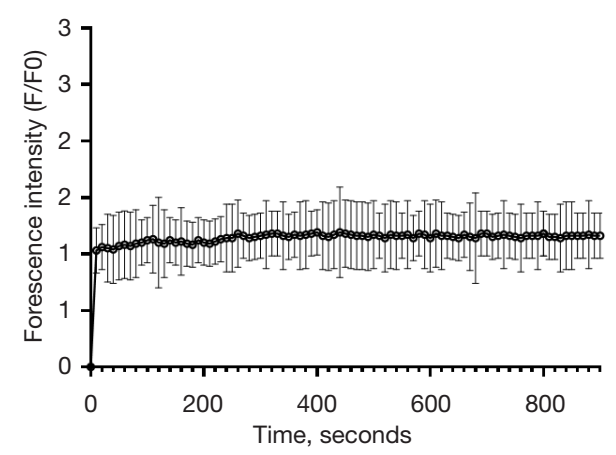

C

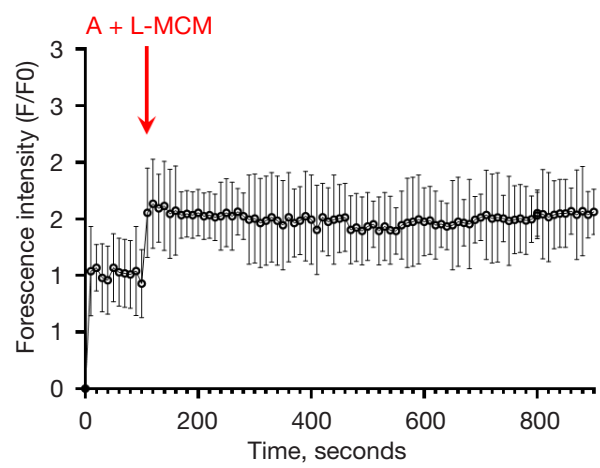

B

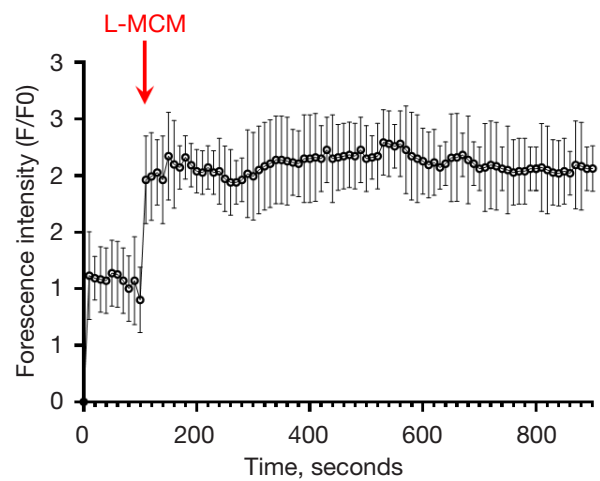

D
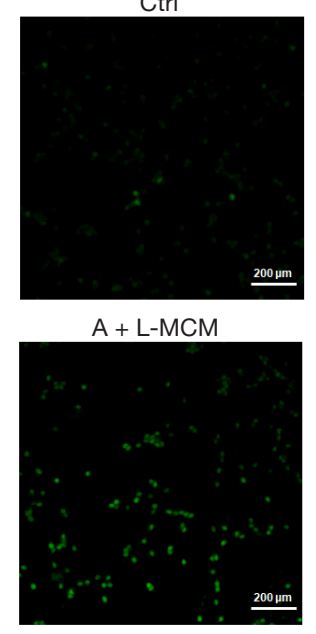
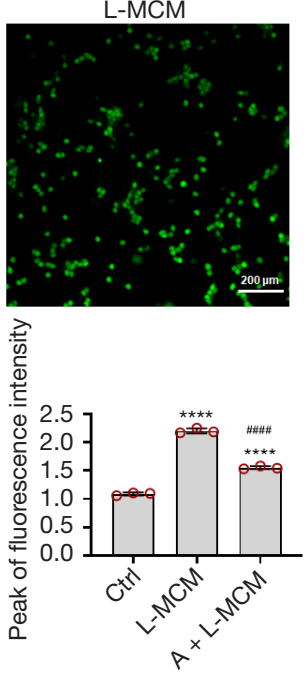

Figure $3 \mathrm{ABPPk}$ pretreatment on microglia reduced the $\mathrm{MCM}$-induced $\mathrm{Ca}^{2+}$ influx into neurons. (A) Baseline of calcium fluorescence signals from cortical neurons without stimulation. (B) Curve of calcium fluorescence amplitude of cortical neurons before and after L-MCM stimulation, with the red arrow indicating the moment of L-MCM stimulation. (C) Curve of calcium fluorescence amplitude of cortical neurons before and after A+L-MCM stimulation, with the red arrow indicating the moment of A+L-MCM stimulation. (D) Representative images of Fluo-4/AM labelled calcium influx fluorescence intensity reaching its peak in cortical neurons and the analysis of peak fluorescence intensity. Scale bar, $200 \mu \mathrm{m}$. Data are presented as the relative fluorescence intensity quantified over 30 single neurons over control and expressed as mean $\pm \mathrm{SD}(\mathrm{n}=3)$. **** $\mathrm{P}<0.0001$ vs. Ctrl; ${ }^{* \# \# \#, ~} \mathrm{P}<0.0001$ vs. L-MCM. Experiment was triplicated biologically. ABPPk, Achyranthes bidentata polypeptide fraction k; MCM, microglia conditioned medium.

concentration-dependently (Figure $8 A$ ). However, when the PI3K inhibitor LY294002 was pre-treated $1 \mathrm{~h}$ before $5 \mu \mathrm{g} / \mathrm{mL} \mathrm{ABPPk}$, the phosphorylation of Akt was significantly decreased in an LY294002 concentrationdependent manner, suggesting that the activation of Akt by ABPPk could be blocked by the PI3K inhibitor (Figure 8B). To further investigate whether PI3K/Akt pathway is crucial for the inhibition of NOX2 by ABPPk, microglia were pretreated with $10 \mu \mathrm{M} \mathrm{LY} 294002$ for $1 \mathrm{~h}$, then $5 \mu \mathrm{g} / \mathrm{mL}$ ABPPk was added in the culture for $30 \mathrm{~min}$, followed by exposure to $1 \mu \mathrm{g} / \mathrm{mL}$ LPS for $24 \mathrm{~h}$. Western blot analysis showed that pretreatment with LY294002 eliminated the inhibition of NOX2 by ABPPk in LPSactivated microglia (Figure $8 C$ ), which resulted in the elimination of ABPPk's inhibitory effect on ROS production and glutamate release induced by LPS (Figure $8 D, 8 E$ ).

In order to further verify the role of PI3K/Akt in NOX2/ ROS signaling pathway, microglia were pretreated with an Akt-specific activator SC79 for $30 \mathrm{~min}$ at a concentration of $4 \mu \mathrm{g} / \mathrm{mL}$, then stimulated with LPS for $24 \mathrm{~h}$. The results showed that SC79 significantly upregulated the expression level of LPS-inhibited phosphorylated Akt, inhibited LPS- 

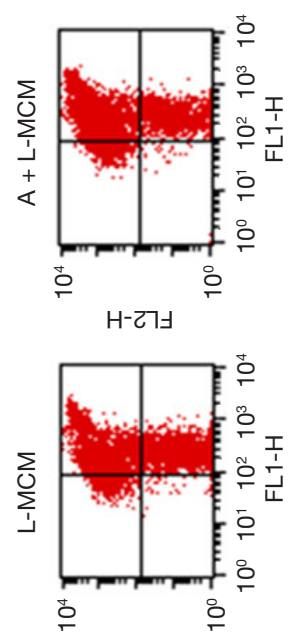

$\mathrm{H}$-乙าษ

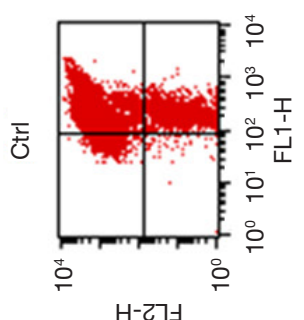

$\infty$
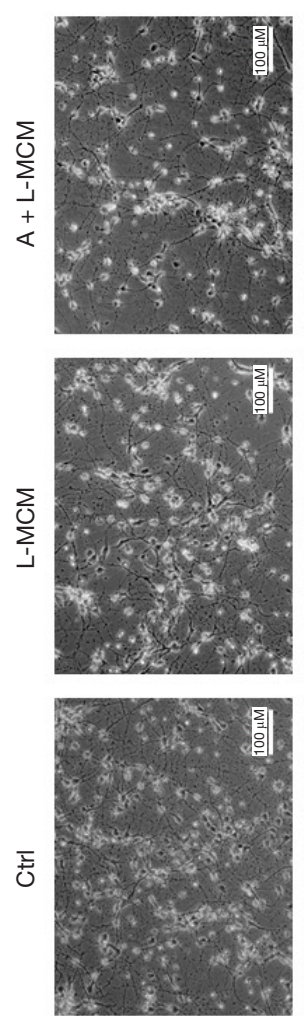

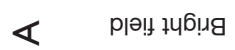
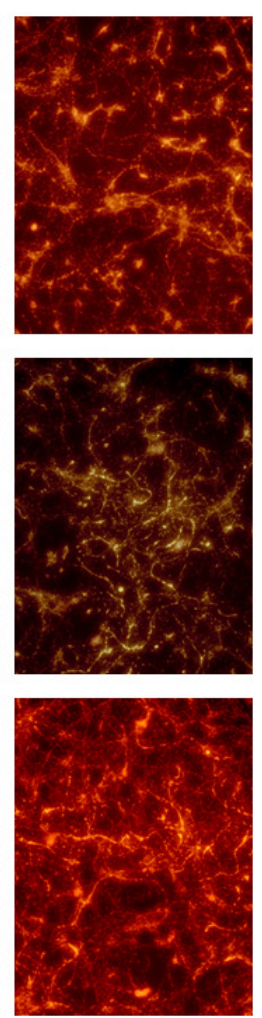

sәңебәл6б
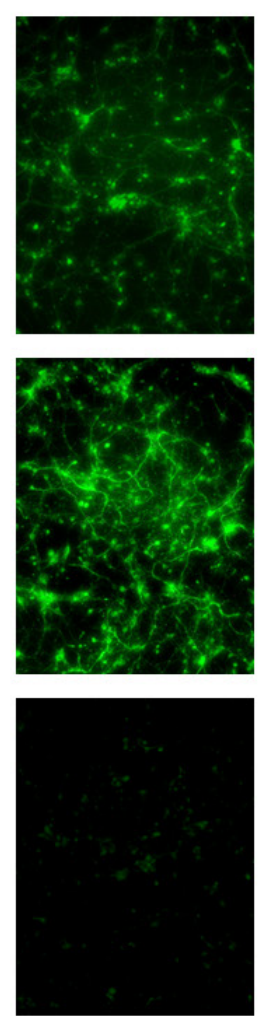

ıәшоиоW
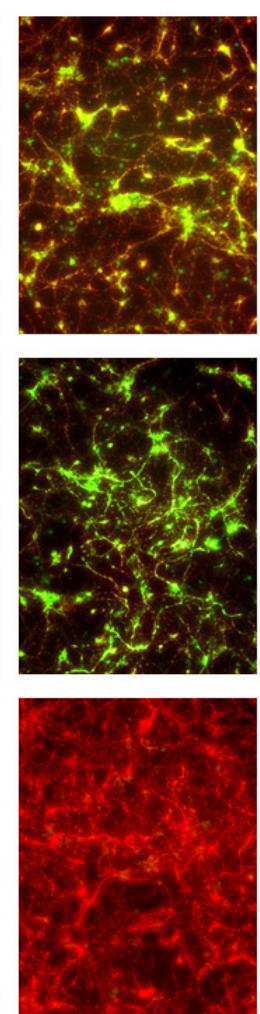

әธิәพ

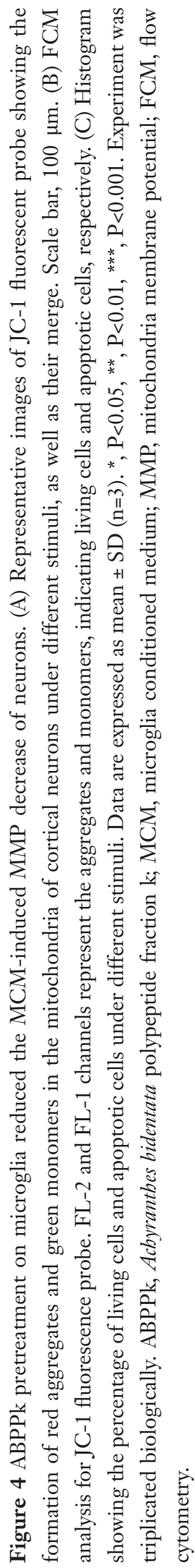

(c) Annals of Translational Medicine. All rights reserved. 
A

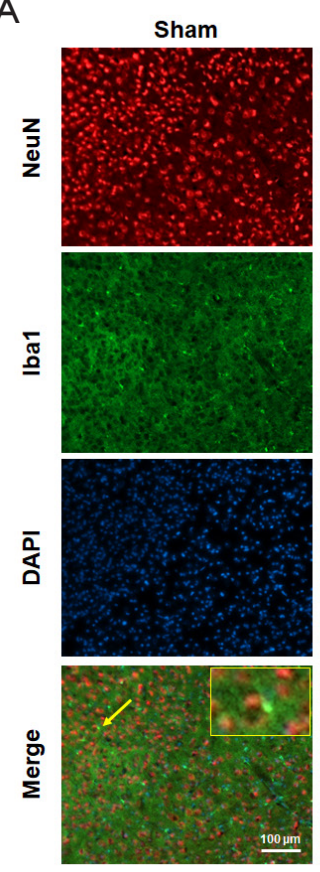

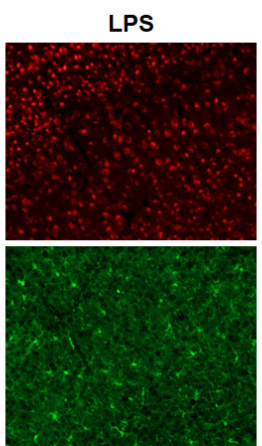
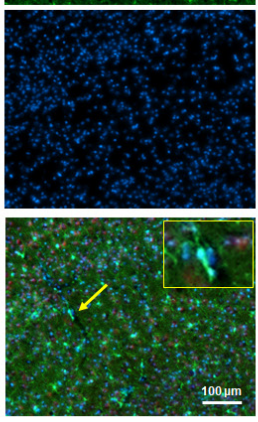

ABPPk
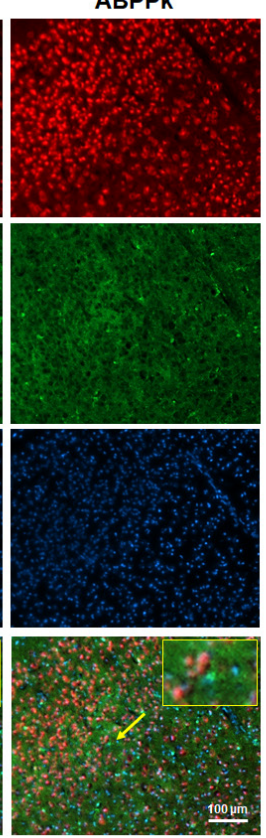

B

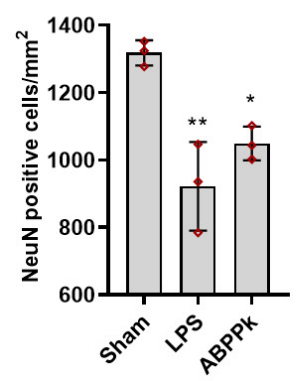

D

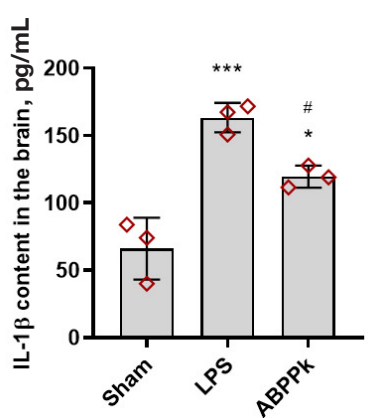

C

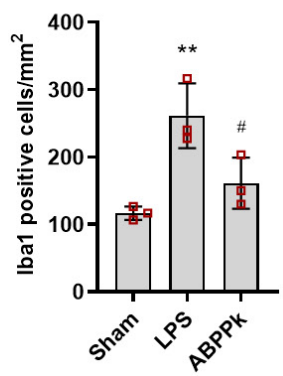

Figure $5 \mathrm{ABPPk}$ alleviated LPS-induced neuronal loss and suppressed microglial activation in the brain. (A) Representative fluorescence images of neurons and microglia in the cortex after LPS intracerebral injection examined by using anti-NeuN (red), anti-Iba1 (green) antibodies and DAPI (blue) as well as their merge. Scale bar, $100 \mu \mathrm{m}$. The yellow box in the upper right corner of the Merge magnifies the part indicated by the arrow. (B) Histogram showing the number of $\mathrm{NeuN}$ positive cells per square millimeter of cortex in each group. Data are expressed as mean $\pm \mathrm{SD}(\mathrm{n}=3)$. ${ }^{*}, \mathrm{P}<0.05,{ }^{* *}, \mathrm{P}<0.01$ vs. Sham. (C) Histogram showing the number of Iba1 positive cells per square millimeter of cortex in each group. Data are expressed as mean $\pm \mathrm{SD}(\mathrm{n}=3)$. ${ }^{* *}, \mathrm{P}<0.01$ vs. Sham;, $\mathrm{P}<0.05$ vs. LPS. (D) Histogram showing the content of IL- $1 \beta$ in the supernatant of brain tissue. Data are expressed as mean $\pm \mathrm{SD}(\mathrm{n}=3) .{ }^{*}, \mathrm{P}<0.05,{ }^{* * *}, \mathrm{P}<0.001 v s$. Sham; ${ }^{*}, \mathrm{P}<0.05 v s$. LPS. ABPPk, Achyranthes bidentata polypeptide fraction k; LPS, lipopolysaccharide.

induced NOX2 upregulation (Figure $8 F$ ) and ROS production (Figure $8 G$ ), thus inhibited glutamate release (Figure $8 H$ ). These results suggested that the activation of PI3K/Akt pathway by ABPPk was involved in the inhibition of NOX2 expression, ROS production, and glutamate release in LPSactivated microglia.

\section{Discussion}

In this study, we pretreated primary microglia with $\mathrm{ABPPk}$, an active polypeptide isolated from traditional Chinese medicine $A$. bidentata, and found it markedly prevented LPS-activated microglial neurotoxicity. ABPPk pretreated MCM significantly alleviated the excitatory neurotoxicity to cortical neurons. Moreover, ABPPk reduced neuronal damage and suppressed microglial activation as well as cell apoptosis induced by LPS intracerebral injection in rat pups. ABPPk greatly activated Akt, thereby inhibiting the expression of NOX2 and reducing the production of ROS, which ultimately led to the reduced glutamate release of activated microglia. However, PI3K specific inhibitor abolished the inhibitory effects of ABPPk on NOX2 expression, ROS production and glutamate release in activated microglia, whereas Akt specific activator can play a similar role to ABPPk in inhibiting LPS-activated NOX2 expression, ROS production and glutamate release. These data suggest that ABPPk can suppress glutamate release from microglia by inhibiting the PI3K/Akt dependent NOX2/ROS pathway, thereby reduce the excitatory neurotoxicity caused by microglial activation.

A. bidentata is a commonly prescribed Chinese medicinal herb. The phytochemical and pharmacological effects of $A$. bidentata have been studied extensively. Saponins and polysaccharides are considered as the main active components of $A$. bidentata in anti-tumor, analgesia, antiinflammatory and immune regulation. The polypeptides 
A
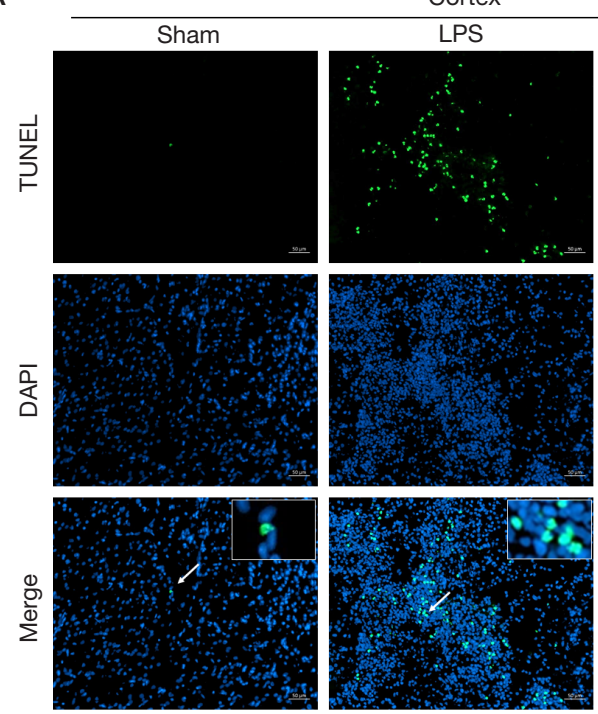
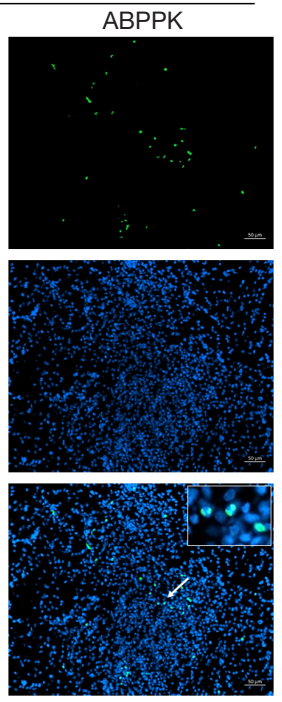
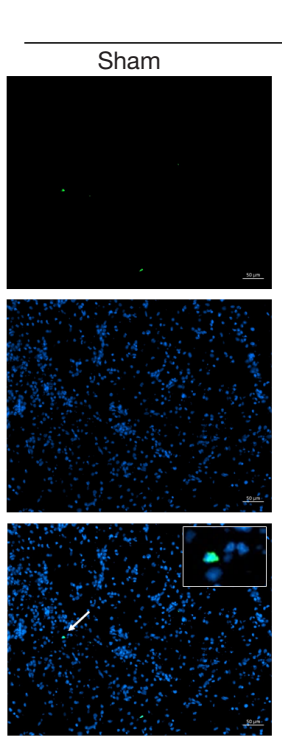
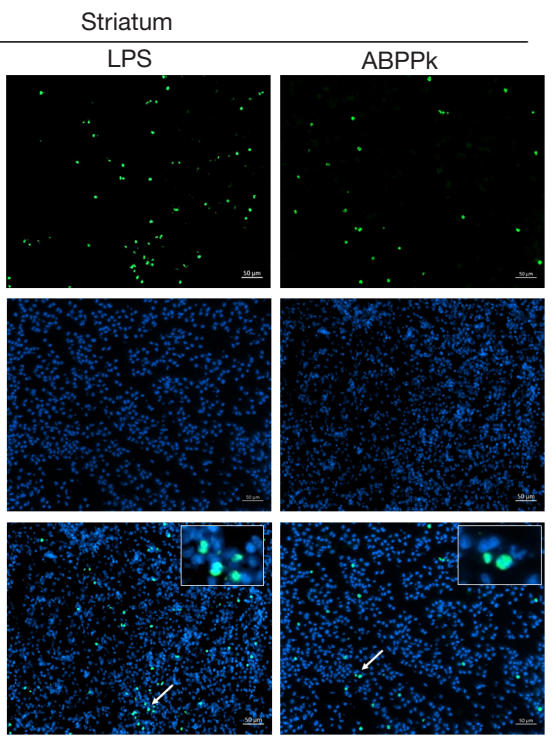

B

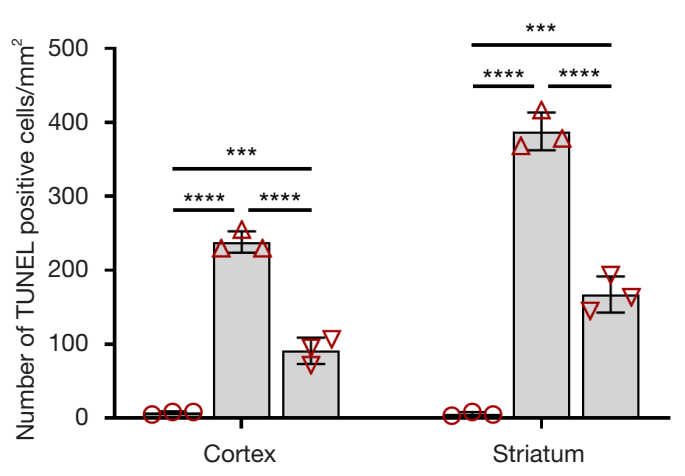

O Sham

$\triangle$ LPS

$\nabla$ ABPPk

Figure 6 ABPPk prevented LPS-induced cell apoptosis in the brain. (A) Representative images of apoptotic cells in the cortex and striatum after LPS intracerebral injection examined by using TUNEL (green) kit and DAPI (blue) as well as their merge. Scale bar, $50 \mu \mathrm{m}$. The white box in the upper right corner of the Merge magnifies the part indicated by the arrow. (B) Histogram showing the number of TUNEL positive cells per square millimeter in the cortex and striatum, respectively. Data are expressed as mean $\pm \mathrm{SD}(\mathrm{n}=3) .{ }^{* * *}, \mathrm{P}<0.001,{ }^{* * * *}$, $\mathrm{P}<0.0001$. ABPPk, Achyranthes bidentata polypeptide fraction k; LPS, lipopolysaccharide.

component extracted from $A$. bidentata exhibited potent neurotrophic and neuroprotective effects, including reducing excitotoxic injury by inhibiting NMDA receptor $(4,22)$, and activating PI3K/Akt pathway to promote neuronal survival under serum deprivation conditions (35). The purified component $\mathrm{k}$ from $A$. bidentata polypeptides (ABPPk) could protect neurons against apoptosis induced by oxygen and glucose deprivation (OGD) and $\mathrm{MMP}^{+}$ insult $(5,6)$. In other studies, ABPPk was reported to protect Schwann cells from oxidative stress-induced apoptosis by activating PI3K/Akt and ERK1/2 signaling pathways (36). We have found that ABPPk could inhibit the inflammatory responses of LPS-activated BV2 microglia (8), however, the specific mechanism of ABPPk's neuroprotective effect by inhibiting neuroinflammation remains unclear.

Normally, microglia are resident brain immune cells, while activated microglia in brain pathologies are key actors of neuroinflammation. Activated microglia may protect neurons by accelerating pathogen clearance and promoting neuronal survival by releasing beneficial nutritional factors (37-39). However, continuously over-activated microglia release large amounts of superoxide, derivative oxidants, NO, TNF- $\alpha$, and glutamate to damage neurons $(40,41)$. The direct neurotoxicity of TNF- $\alpha$ secreted by 
A
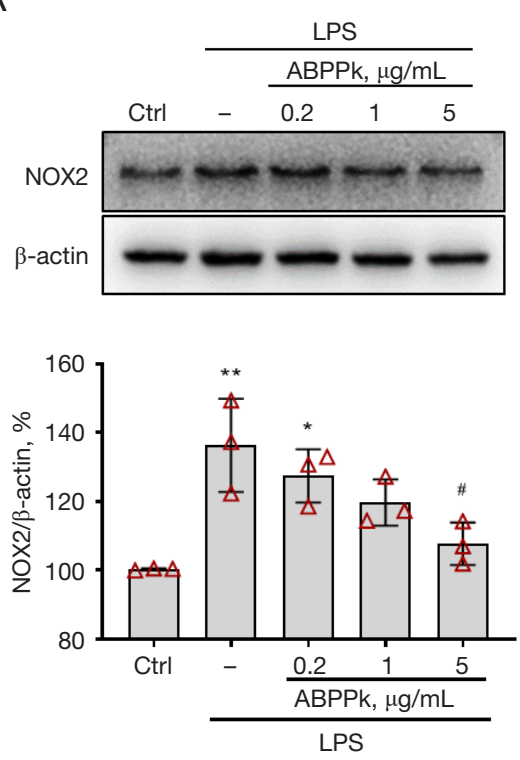

B

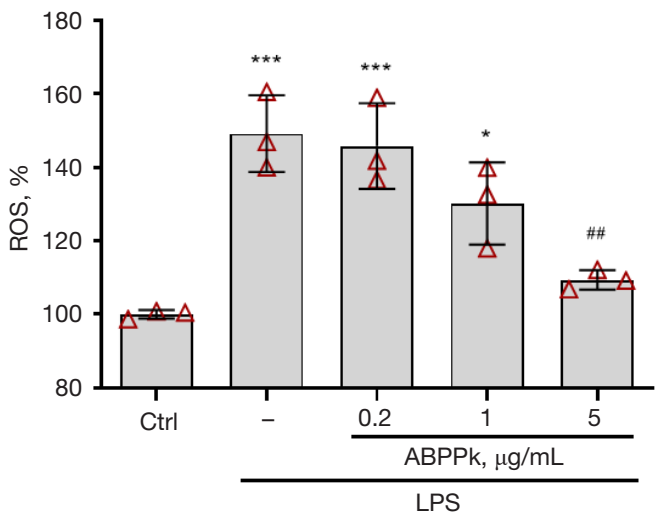

Figure 7 ABPPk inhibited LPS-induced NOX2 expression and ROS production in microglia. (A) Representative Western blot image and gray analysis showing the NOX2 expression in different treated cultures as indicated. Data are expressed as mean $\pm \mathrm{SD}(\mathrm{n}=3)$. *, $\mathrm{P}<0.05$, **, $\mathrm{P}<0.01$ vs. Ctrl; \#, $\mathrm{P}<0.05$ vs. LPS. (B) Histogram showing the ROS production measured by DCFH-DA. Data are expressed as mean \pm SD (n=3). * $\mathrm{P}<0.05,{ }^{* * *}, \mathrm{P}<0.001$ vs. Ctrl; ${ }^{\#}, \mathrm{P}<0.01$ vs. LPS. Experiments were triplicated biologically. ABPPk, Achyranthes bidentata polypeptide fraction k; LPS, lipopolysaccharide; ROS, reactive oxygen species.

activated microglia is relatively weak, but it can stimulate extensive glutamate release from microglia to induce direct excitotoxicity and aggravate neuronal injury $(15,42)$. High levels of NO may promote glutamate release and activate the glutamate NMDA receptor, which amplifies the neurotoxicity of glutamate $(43,44)$. Thus, glutamate is the key determinant inducing excitoneurotoxicity $(17,18)$. Elevated levels of extracellular glutamate will stimulate neuronal NMDA receptors resulting in uncontrolled $\mathrm{Ca}^{2+}$ increases in most neurons (45-47). In fact, glutamate excitotoxicity is thought to be the ultimate common pathway for many neurological diseases, including stroke, Huntington's disease, the acquired immunodeficiency syndrome (AIDS) dementia, and amyotrophic lateral sclerosis (ALS) (48).

Previously we have demonstrated that ABPPk pretreatment could inhibit the LPS-induced increase of TNF- $\alpha$ and NO in BV2 microglia, suggesting the antiinflammatory effect of ABPPk (8). Assembly of NLRP3 inflammasome occurs in activated microglia, leading to cleavage and increased activity of caspase 1 , and release of downstream IL-1 $\beta$ and IL-18, which are critical links in microglial inflammation (49). In this study, ABPPk pretreatment inhibited LPS-induced upregulation of NLRP3 and cleaved caspase 1 in primary microglia as well as the mRNA levels of IL- $1 \beta$ and IL-18 in a concentration dependent manner. The excitatory toxicity of MCM collected from ABPPk pretreated microglia was significantly lower than that of MCM without ABPPk pretreatment. In vivo results also showed that $\mathrm{ABPPk}$ can effectively inhibit the activation of microglia induced by LPS intracerebral injection, and reduce cell apoptosis. Notably, $\mathrm{ABPPk}$ pretreatment resulted in a concentration-dependent reduction in glutamate release from LPS-activated microglia. These results suggested that ABPPk can inhibit the neurotoxicity of activated microglia, and the direct inhibition of glutamate release from activate microglia may be a very important link.

Glutamate release is closely related to oxidative stress in activated microglia (50). In other words, ROS, such as lipid peroxidation products, superoxide, and derivative oxidants, are precisely the preconditions for glutamate release (19). LPS can lead to classical activation of microglia and upregulate the expression of NADPH oxidase, especially NOX2 transmembrane subunit to generate a large number of ROS, driving oxidative stress $(51,52)$. 
A

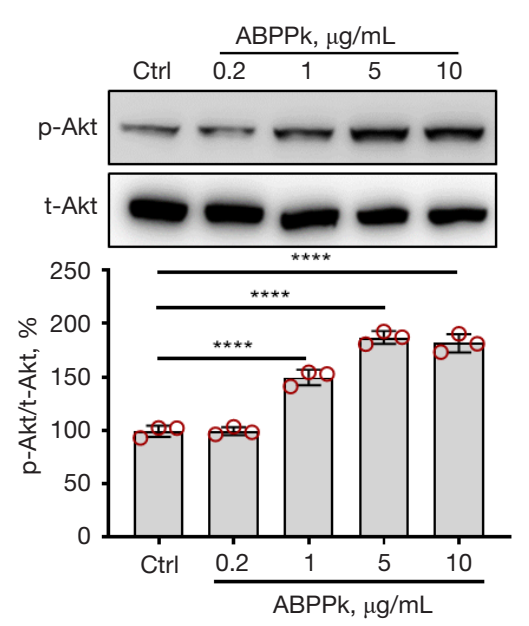

D

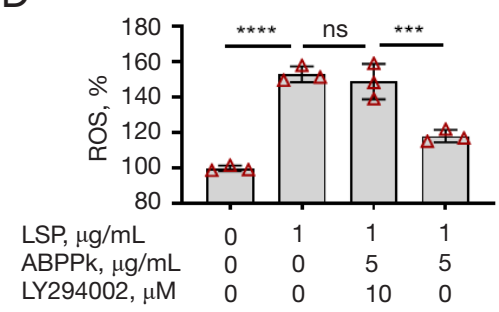

$E$

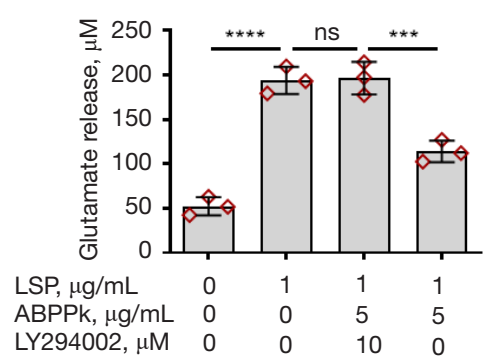

B

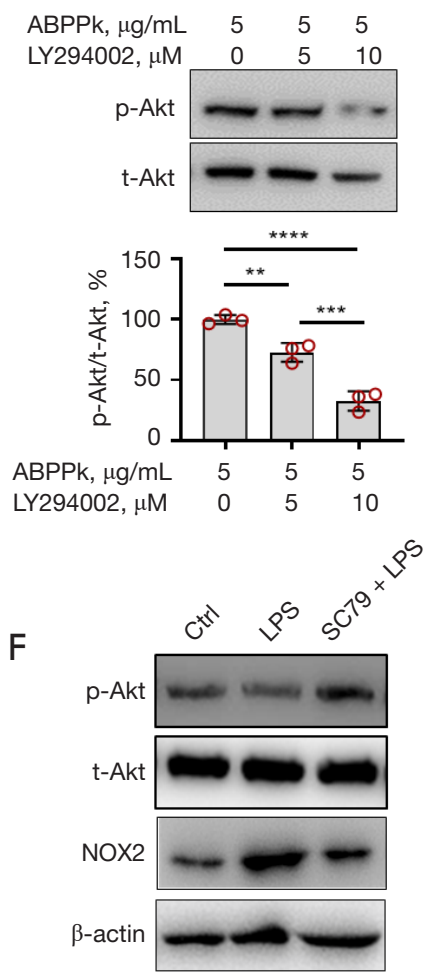

C

$\begin{array}{lllll}\text { LSP, } \mu \mathrm{g} / \mathrm{mL} & 0 & 1 & 1 & 1 \\ \text { ABPPk, } \mu \mathrm{g} / \mathrm{mL} & 0 & 0 & 5 & 5 \\ \text { LY294002, } \mu \mathrm{M} & 0 & 0 & 10 & 0\end{array}$

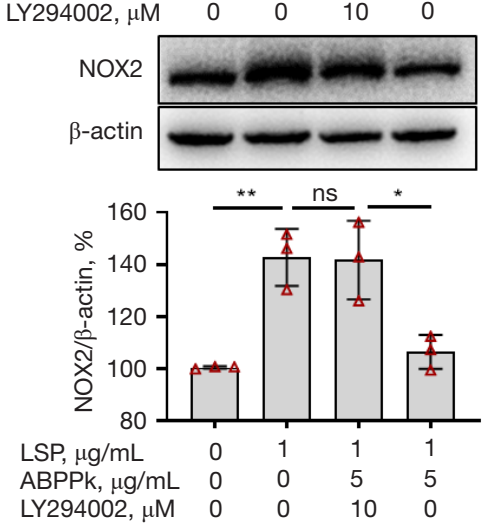

G

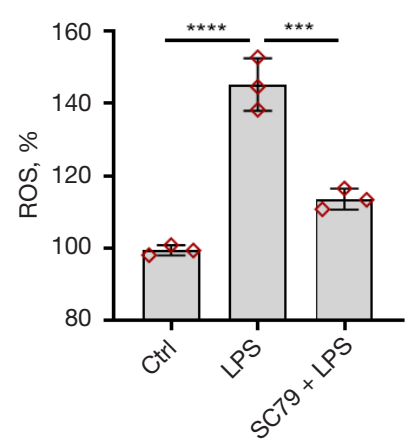

$\mathrm{H}$

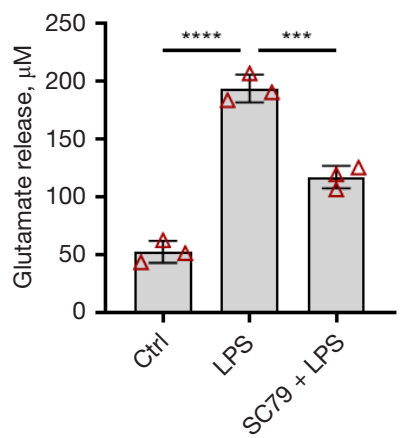

Figure 8 ABPPk inhibited LPS-induced glutamate release of microglia via PI3K/Akt dependent NOX2/ROS pathway. (A) Representative Western blot image and gray analysis showing phosphorylated Akt (p-Akt) expression levels after treatment with indicated concentrations of ABPPk for $30 \mathrm{~min}$. Data are expressed as mean $\pm \mathrm{SD}(\mathrm{n}=3) .{ }^{* * *}, \mathrm{P}<0.0001$. (B) Representative Western blot image and gray analysis showing phosphorylated Akt (p-Akt) expression levels after treatment with $5 \mu \mathrm{g} / \mathrm{mL}$ ABPPk for $30 \mathrm{~min}$ with or without LY294002 pretreatment. Data are expressed as mean $\pm \mathrm{SD}(\mathrm{n}=3) .{ }^{* *}, \mathrm{P}<0.01,{ }^{* * *}, \mathrm{P}<0.001,{ }^{* * * *}, \mathrm{P}<0.0001$. (C) Representative Western blot image and gray analysis showing the NOX2 expression in microglia treated as indicated. Data are expressed as mean $\pm \mathrm{SD}(\mathrm{n}=3)$. * $, \mathrm{P}<0.05,{ }^{* *}, \mathrm{P}<0.01$, and ns means no significance. (D) Histogram showing the impact of indicated treatment on ROS production in activated microglia. Data are expressed as mean $\pm \mathrm{SD}(\mathrm{n}=3) .{ }^{* * *}, \mathrm{P}<0.001,{ }^{* * * *}, \mathrm{P}<0.0001$, and ns means no significance. (E) Histogram showing the impact of indicated treatment on glutamate release in activated microglia. Data are expressed as mean $\pm \mathrm{SD}(\mathrm{n}=3)$. ${ }^{* * *}, \mathrm{P}<0.001$, ${ }^{* * *}, \mathrm{P}<0.0001$, and ns means no significance. (F) Representative Western blot image and gray analysis showing phosphorylated Akt (p-Akt) and NOX2 expression levels after SC79 pretreatment in LPS-activated microglia. Data are expressed as mean \pm SD $(\mathrm{n}=3) .{ }^{*}, \mathrm{P}<0.05,{ }^{* *}, \mathrm{P}<0.01,{ }^{* * *}, \mathrm{P}<0.001$. (G) Histogram showing the effect of $\mathrm{SC} 79$ pretreatment on ROS production in LPS-activated microglia. Data are expressed as mean $\pm \mathrm{SD}(\mathrm{n}=3) .{ }^{* * *}, \mathrm{P}<0.001,{ }^{* * * *}, \mathrm{P}<0.0001$. (H) Histogram showing the effect of SC79 pretreatment on glutamate release in LPS-activated microglia. Data are expressed as mean $\pm \mathrm{SD}(\mathrm{n}=3) .{ }^{* * *}, \mathrm{P}<0.001,{ }^{* * * *}, \mathrm{P}<0.0001$. Experiments were triplicated biologically. ABPPk, Achyranthes bidentata polypeptide fraction k; LPS, lipopolysaccharide; ROS, reactive oxygen species. 
NOX2 is the main isoform of NADPH oxidase, also known as gp91phox, which is most abundant in microglia $(32,53)$. NOX2 directly catalyzes ROS biosynthesis, a process known as oxidative burst (54), which is accompanied by damage of redox signal transduction and mitochondrial dysfunction (55). During early inflammation, polarization to M1 phenotype microglia is dependent on NOX2 activation (56), and NOX2-derived ROS could inhibit the activation of PI3K/Akt survival pathway (57).

The activation of PI3K/Akt can promote the shift from pro-inflammatory M1 phenotype to anti-inflammatory M2 phenotype in LPS-stimulated microglia (58,59). OGD insult can induce ROS production by upregulating NOX2 via the PI3K/Akt-dependent NF- $\kappa \mathrm{B}$ signaling pathway, suggesting that activation of PI3K/Akt may be beneficial to inhibit NOX2 expression and ROS production (60). The specific Akt activator, SC79, has been reported to protect neuronal cells from $\mathrm{MPP}^{+}$or ischemic injury $(61,62)$. It has also been proved that SC79 can abolish the inhibitory effect of NOX2 inhibitors on $\mathrm{O}_{2}{ }^{-}$production in hyperhomocysteinemia models, which confirmed that PI3K/Akt pathway is critically involved in the NOX2/ROS signaling transduction (63). In this present study, $\mathrm{ABPPk}$ pretreatment concentrationdependently inhibited LPS-induced NOX2 expression in microglia. Moreover, ABPPk significantly upregulated Akt phosphorylation in a concentration-dependent manner, and this effect could be eliminated by the PI3K specific inhibitor LY294002. Furthermore, the inhibitory effect of ABPPk on LPS-upregulated NOX2 expression was also eliminated by LY294002. Meanwhile, pretreatment with SC79 followed by LPS stimulation increased the phosphorylation of Akt and inhibited the expression of NOX2. As expected, LY294002 also abolished the inhibitory effect of ABPPk on LPS-induced ROS production and glutamate release, while SC79 played a role similar to that of ABPPk, namely inhibiting LPS-induced ROS production and glutamate release from microglia.

Taken together, ABPPk attenuates the microgliaderived excitatory neurotoxicity by inhibiting the PI3K/ Akt-dependent NOX2/ROS pathway, which might provide new sight for the intervention and therapy of neuroinflammation.

There are some limitations in our study. ABPPk, as a polypeptide fraction of plant origin, is likely to be multitarget, and transcriptomic and proteomic studies may help to clarify its mechanisms in the future.

\section{Acknowledgments}

Funding: This research was supported by grants from the National Natural Science Foundation of China, No. 81501058, 82071355; the Priority Academic Program Development of Jiangsu Higher Education Institutions (PAPD), No. 20KJA310011, and a grant from Science and Technology Project of Nantong City, No. JC2020001.

\section{Footnote}

Reporting Checklist: The authors have completed the MDAR reporting checklist. Available at https://dx.doi. org/10.21037/atm-21-4027

Data Sharing Statement: Available at https://dx.doi. org/10.21037/atm-21-4027

Conflicts of Interest: All authors have completed the ICMJE uniform disclosure form (available at https://dx.doi. org/10.21037/atm-21-4027). The authors have no conflicts of interest to declare.

Ethical Statement: The authors are accountable for all aspects of the work in ensuring that questions related to the accuracy or integrity of any part of the work are appropriately investigated and resolved. The study was approved by the Administration Committee of Experimental Animals of Nantong University. All the experimental procedures were performed according to the Chinese Guidelines for the Care and Use of Laboratory Animals.

Open Access Statement: This is an Open Access article distributed in accordance with the Creative Commons Attribution-NonCommercial-NoDerivs 4.0 International License (CC BY-NC-ND 4.0), which permits the noncommercial replication and distribution of the article with the strict proviso that no changes or edits are made and the original work is properly cited (including links to both the formal publication through the relevant DOI and the license). See: https://creativecommons.org/licenses/by-nc-nd/4.0/.

\section{References}

1. He X, Wang X, Fang J, et al. The genus Achyranthes: A review on traditional uses, phytochemistry, and 
pharmacological activities. J Ethnopharmacol 2017;203:260-78.

2. Jin LQ, Zheng ZJ, Peng Y, et al. Opposite effects on tumor growth depending on dose of Achyranthes bidentata polysaccharides in C57BL/6 mice. Int Immunopharmacol 2007;7:568-77.

3. Zhou S, Chen X, Gu X, et al. Achyranthes bidentata Blume extract protects cultured hippocampal neurons against glutamate-induced neurotoxicity. J Ethnopharmacol 2009;122:547-54.

4. Shen H, Yuan Y, Ding F, et al. Achyranthes bidentata polypeptides confer neuroprotection through inhibition of reactive oxygen species production, Bax expression, and mitochondrial dysfunction induced by overstimulation of $\mathrm{N}$-methyl-D-aspartate receptors. J Neurosci Res 2010;88:669-76.

5. Yu S, Wang C, Cheng Q, et al. An active component of Achyranthes bidentata polypeptides provides neuroprotection through inhibition of mitochondrialdependent apoptotic pathway in cultured neurons and in animal models of cerebral ischemia. PLoS One 2014;9:e109923.

6. Peng S, Wang C, Ma J, et al. Achyranthes bidentata polypeptide protects dopaminergic neurons from apoptosis in Parkinson's disease models both in vitro and in vivo. $\mathrm{Br}$ J Pharmacol 2018;175:631-43.

7. Cheng Q, Tong F, Shen Y, et al. Achyranthes bidentata polypeptide $\mathrm{k}$ improves long-term neurological outcomes through reducing downstream microvascular thrombosis in experimental ischemic stroke. Brain Res 2019;1706:166-76.

8. Cheng Q, Shen Y, Cheng Z, et al. Achyranthes bidentata polypeptide $\mathrm{k}$ suppresses neuroinflammation in BV2 microglia through Nrf2-dependent mechanism. Ann Transl Med 2019;7:575.

9. Colton CA. Heterogeneity of microglial activation in the innate immune response in the brain. J Neuroimmune Pharmacol 2009;4:399-418.

10. Boche D, Perry VH, Nicoll JA. Review: activation patterns of microglia and their identification in the human brain. Neuropathol Appl Neurobiol 2013;39:3-18.

11. Ransohoff RM, Brown MA. Innate immunity in the central nervous system. J Clin Invest 2012;122:1164-71.

12. Hickman S, Izzy S, Sen P, et al. Microglia in neurodegeneration. Nat Neurosci 2018;21:1359-69.

13. Hanisch UK, Kettenmann H. Microglia: active sensor and versatile effector cells in the normal and pathologic brain. Nat Neurosci 2007;10:1387-94.
14. Takeuchi H, Mizuno T, Zhang G, et al. Neuritic beading induced by activated microglia is an early feature of neuronal dysfunction toward neuronal death by inhibition of mitochondrial respiration and axonal transport. J Biol Chem 2005;280:10444-54.

15. Takeuchi H, Jin S, Wang J, et al. Tumor necrosis factoralpha induces neurotoxicity via glutamate release from hemichannels of activated microglia in an autocrine manner. J Biol Chem 2006;281:21362-8.

16. Mizuno T, Zhang G, Takeuchi H, et al. Interferon-gamma directly induces neurotoxicity through a neuron specific, calcium-permeable complex of IFN-gamma receptor and AMPA GluR1 receptor. FASEB J 2008;22:1797-806.

17. Takeuchi H, Jin S, Suzuki H, et al. Blockade of microglial glutamate release protects against ischemic brain injury. Exp Neurol 2008;214:144-6.

18. Suzumura A. Neuron-microglia interaction in neuroinflammation. Curr Protein Pept Sci 2013;14:16-20.

19. Barger SW, Goodwin ME, Porter MM, et al. Glutamate release from activated microglia requires the oxidative burst and lipid peroxidation. J Neurochem 2007;101:1205-13.

20. Panfoli I, Candiano G, Malova M, et al. Oxidative Stress as a Primary Risk Factor for Brain Damage in Preterm Newborns. Front Pediatr 2018;6:369.

21. Yang Q, Huang Q, Hu Z, et al. Potential Neuroprotective Treatment of Stroke: Targeting Excitotoxicity, Oxidative Stress, and Inflammation. Front Neurosci 2019;13:1036.

22. Shen H, Yuan Y, Ding F, et al. The protective effects of Achyranthes bidentata polypeptides against NMDAinduced cell apoptosis in cultured hippocampal neurons through differential modulation of NR2A- and NR2B-containing NMDA receptors. Brain Res Bull 2008;77:274-81.

23. Sepulveda-Diaz JE, Ouidja MO, Socias SB, et al. A simplified approach for efficient isolation of functional microglial cells: Application for modeling neuroinflammatory responses in vitro. Glia 2016;64:1912-24.

24. Sciarretta C, Minichiello L. The preparation of primary cortical neuron cultures and a practical application using immunofluorescent cytochemistry. Methods Mol Biol 2010;633:221-31.

25. Urrutia PJ, Hirsch EC, González-Billault C, et al. Hepcidin attenuates amyloid beta-induced inflammatory and pro-oxidant responses in astrocytes and microglia. J Neurochem 2017;142:140-52.

26. Fan LW, Mitchell HJ, Rhodes PG, et al. Alpha-Phenyl-n- 
tert-butyl-nitrone attenuates lipopolysaccharide-induced neuronal injury in the neonatal rat brain. Neuroscience 2008;151:737-44.

27. Tien LT, Kaizaki A, Pang Y, et al. Neonatal exposure to lipopolysaccharide enhances accumulation of $\alpha$-synuclein aggregation and dopamine transporter protein expression in the substantia nigra in responses to rotenone challenge in later life. Toxicology 2013;308:96-103.

28. Grin'kina NM, Karnabi EE, Damania D, et al. Sphingosine kinase 1 deficiency exacerbates LPS-induced neuroinflammation. PLoS One 2012; 7:e36475.

29. Martinon F, Burns K, Tschopp J. The inflammasome: a molecular platform triggering activation of inflammatory caspases and processing of proIL-beta. Mol Cell 2002;10:417-26.

30. Sutterwala FS, Ogura Y, Szczepanik M, et al. Critical role for NALP3/CIAS1/Cryopyrin in innate and adaptive immunity through its regulation of caspase-1. Immunity 2006;24:317-27.

31. Tehse J, Taghibiglou C. The overlooked aspect of excitotoxicity: Glutamate-independent excitotoxicity in traumatic brain injuries. Eur J Neurosci 2019;49:1157-70.

32. Bedard K, Krause KH. The NOX family of ROSgenerating NADPH oxidases: physiology and pathophysiology. Physiol Rev 2007;87:245-313.

33. Lee WJ, Ham SA, Yoo H, et al. Activation of PPAR $\delta$ attenuates neurotoxicity by inhibiting lipopolysaccharidetriggered glutamate release in BV-2 microglial cells. J Cell Biochem 2018;119:5609-19.

34. Zhang HS, Sang WW, Ruan Z, et al. Akt/Nox2/NF-кB signaling pathway is involved in Tat-induced HIV-1 long terminal repeat (LTR) transactivation. Arch Biochem Biophys 2011;505:266-72.

35. Shen $Y$, Zhang Q, Gao X, et al. An active fraction of Achyranthes bidentata polypeptides prevents apoptosis induced by serum deprivation in SH-SY5Y cells through activation of PI3K/Akt/Gsk3 $\beta$ pathways. Neurochem Res 2011;36:2186-94.

36. Li M, Zhu Y, Peng W, et al. Achyranthes bidentata Polypeptide Protects Schwann Cells From Apoptosis in Hydrogen Peroxide-Induced Oxidative Stress. Front Neurosci 2018;12:868.

37. Haukedal H, Freude K. Implications of Microglia in Amyotrophic Lateral Sclerosis and Frontotemporal Dementia. J Mol Biol 2019;431:1818-29.

38. Gupta N, Shyamasundar S, Patnala R, et al. Recent progress in therapeutic strategies for microglia-mediated neuroinflammation in neuropathologies. Expert Opin
Ther Targets 2018;22:765-81.

39. Loane DJ, Kumar A. Microglia in the TBI brain: The good, the bad, and the dysregulated. Exp Neurol 2016;275 Pt 3:316-27.

40. Ransohoff RM. How neuroinflammation contributes to neurodegeneration. Science 2016;353:777-83.

41. Brown GC, Vilalta A. How microglia kill neurons. Brain Res 2015;1628:288-97.

42. Chen CJ, Ou YC, Chang CY, et al. Glutamate released by Japanese encephalitis virus-infected microglia involves TNF- $\alpha$ signaling and contributes to neuronal death. Glia 2012;60:487-501

43. Bal-Price A, Brown GC. Inflammatory neurodegeneration mediated by nitric oxide from activated glia-inhibiting neuronal respiration, causing glutamate release and excitotoxicity. J Neurosci 2001;21:6480-91.

44. Yuste JE, Tarragon E, Campuzano CM, et al. Implications of glial nitric oxide in neurodegenerative diseases. Front Cell Neurosci 2015;9:322.

45. Ye ZC, Sontheimer H. Glioma cells release excitotoxic concentrations of glutamate. Cancer Res 1999;59:4383-91.

46. Olney JW, de Gubareff T. Glutamate neurotoxicity and Huntington's chorea. Nature 1978;271:557-9.

47. Choi DW. Glutamate neurotoxicity and diseases of the nervous system. Neuron 1988;1:623-34.

48. Lipton SA, Rosenberg PA. Excitatory amino acids as a final common pathway for neurologic disorders. N Engl J Med 1994;330:613-22.

49. Thawkar BS, Kaur G. Inhibitors of NF- $\mathrm{B}$ and $\mathrm{P} 2 \mathrm{X} 7 /$ NLRP3/Caspase 1 pathway in microglia: Novel therapeutic opportunities in neuroinflammation induced early-stage Alzheimer's disease. J Neuroimmunol 2019;326:62-74.

50. Batista CRA, Gomes GF, Candelario-Jalil E, et al. Lipopolysaccharide-Induced Neuroinflammation as a Bridge to Understand Neurodegeneration. Int J Mol Sci 2019;20:2293

51. Qin L, Liu Y, Hong JS, et al. NADPH oxidase and aging drive microglial activation, oxidative stress, and dopaminergic neurodegeneration following systemic LPS administration. Glia 2013;61:855-68.

52. Colton C, Wilcock DM. Assessing activation states in microglia. CNS Neurol Disord Drug Targets 2010;9:174-91.

53. Royer-Pokora B, Kunkel LM, Monaco AP, et al. Cloning the gene for an inherited human disorder--chronic granulomatous disease--on the basis of its chromosomal location. Nature 1986;322:32-8. 
54. Sareila O, Kelkka T, Pizzolla A, et al. NOX2 complexderived ROS as immune regulators. Antioxid Redox Signal 2011;15:2197-208.

55. Green SP, Cairns B, Rae J, et al. Induction of gp91phox, a component of the phagocyte NADPH oxidase, in microglial cells during central nervous system inflammation. J Cereb Blood Flow Metab 2001;21:374-84.

56. Griffiths HR, Gao D, Pararasa C. Redox regulation in metabolic programming and inflammation. Redox Biol 2017;12:50-7.

57. Ghoshal P, Singla B, Lin H, et al. Nox2-Mediated PI3K and Cofilin Activation Confers Alternate Redox Control of Macrophage Pinocytosis. Antioxid Redox Signal 2017;26:902-16.

58. Tarassishin L, Suh HS, Lee SC. Interferon regulatory factor 3 plays an anti-inflammatory role in microglia by activating the PI3K/Akt pathway. J Neuroinflammation 2011;8:187.

59. Chen S, Peng J, Sherchan P, et al. TREM2 activation

Cite this article as: Wang Y, Ge X, Yu S, Cheng Q. Achyranthes bidentata polypeptide alleviates neurotoxicity of lipopolysaccharide-activated microglia via PI3K/Akt dependent NOX2/ROS pathway. Ann Transl Med 2021;9(20):1522. doi: 10.21037/atm-21-4027 attenuates neuroinflammation and neuronal apoptosis via PI3K/Akt pathway after intracerebral hemorrhage in mice. J Neuroinflammation 2020;17:168.

60. Chern CM, Liou KT, Wang YH, et al. Andrographolide inhibits PI3K/AKT-dependent NOX2 and iNOS expression protecting mice against hypoxia/ ischemia-induced oxidative brain injury. Planta Med 2011;77:1669-79.

61. Zhu JL, Wu YY, Wu D, et al. SC79, a novel Akt activator, protects dopaminergic neuronal cells from $\mathrm{MPP}+$ and rotenone. Mol Cell Biochem 2019;461:81-9.

62. Luan Q, Pan L, He D, et al. SC79, the AKT Activator Protects Cerebral Ischemia in a Rat Model of Ischemia/ Reperfusion Injury. Med Sci Monit 2018;24:5391-7.

63. Smith RM, Rai S, Kruzliak P, et al. Putative Nox2 inhibitors worsen homocysteine-induced impaired acetylcholine-mediated relaxation. Nutr Metab Cardiovasc Dis 2019;29:856-64. 